\title{
Die Behandlung der Syphilis im Lichte der neueren Syphilisforschung.
}

Von Dr. Fritz Lesser, Leiter des serologisehen Laboratoriums.

Wenn wir uns fragen, inwieweit die drei großen Entdeckungen auf dem Gebiete der Syphilis auf die Behandlung dieser Krankheit fördernd gewirkt haben, so haben sich aus der Uebertragbarkeit der Syphilis a uf Tiere zurzeit keinerlei Vorteile für die Behandlung ergeben. Da der Verlauf der tierischen Syphilis andersartig, besonders nicht so chronisch ist und auch die Toleranz gegenüber den Antisyphilitica bei Tier und Mensch verschieden ist, lassen sich doch die aus der tierischen Syphilis gewonnenen Erfahrungen bezïglich der Behandlung nicht leicht auf den Menschen übertragen. Immerhin hat uns die Tiersyphilis nijt dem Atoxyl als Antisyphiliticum bekannt gemacht, und dieses sowie andere Arsenpräparate liaben mehr als frïher bei der Behandlung der menschlichen Syphilis Beachtung gefunden, wenngleich das Quecksilber nach wie vor die erste Stelle einnimmt, sodak alle anderen Mittel nicht als Ersatz-, sondern nur als Ergänzungsmittel zu betracliten sind.

Auch die Entdeckung des Sypliiliserregers hat zurzeit für die Behandlung der Syphilis, so sonderbar es klingen mag, keinerlei praktische Bedeutung. Der Nachweis der Spirochaete pallida ermöglicht zwar schon ganz früh, die Primäreffloreszenz als syphilitisch zu verifizieren, und es könnte a priori scheinen, als ob eine schon vor Ausbildung der Jnduration und indolenter Bubonen eingeleitete antisyphilitische Kur vielleicht bessere Resultate zeitigen würde, quasi eine Abortivheilung der Syphilis ermöglichen könute. Ich habe in einer kleinen Anzalıl von Fällen, wo die Primäreffloreszenz nur durch den Nachweis der Spirochäten als syphilitisch erkannt werden konnte, wo also die Induration, die indolenten Bubonen und auch die positive Wassermannsche Reaktion noch fehlten, die antisyphilitische Kur eingeleitet, energisch durchigeführt und konnte doch nicht verhindern, daß kurze Zeit nach Beendigung derselben das Serum in allen Fällen nach Wassermann positiv reagierte, ja sogar in den meisten Fällen (unter acht Fällen fünfmal) die Roseola zum Ausbruch kam. Daher ist es begreiflich, wenn der alte Standpunkt, erst nach Ausbruch der Roseola nit der Allgemeinbehandlung zu beginnen, wofern nicht besondere Lokalisation des Primäraffektes oder Komplikationen eine sofortige Kur wünschenswert machen, auch heute noch vertreten wird.

Weit fruchtbringender dagegen scheint sich für dic Behandlung der Syphilis die jüngste Errungenschaft, dit serodiagnostik durch Wassermann, zu gestalten. Wenn man der Wassermannschen Reaktion eine Bedeutung für die Behandlung der Syphilis zuschreiben bzw. sie als Maßstab füi die Beurteilung therapeutischer Erfolge gelten lassen will, so ist dabei Voraussetzung, daß eine positive Reaktion nicht bloß besagt, daß der Betreffende mal Syphilis gehabt hat, sondern daß sie als Zeichen noch bestehender Syphilis, d. h. aktiver Spirochäten anzusehen ist; und daß anderseits eine negative Reaktion einen Schluß auf eventuelle Ausheilung der Syphilis zuläßt. Wir sind auf diese Kardinalfrage in der ganzen Serodiagnostik der Syphilis bereits in frülıeren Arbeiten ${ }^{1}$ ) ausführlicher eingegangen und wollen heute nur kurz resünieren, welche Argumente dafür sprechen und zu der Schlußfolgerung berechtigen, daß eine positive Serumreaktion stets noch aktive Syphilis beweist:

1. Die durch pathologiseh-anatomische und sero-

1) Medizinische Klinik 1908, No. 9 und Deutsche medizinische Wochenschrift 1909, No. 9. 
logische Untersuchungen unabhängig voneinander gefundenen, ïbereinstimmenden Prozentzahlen. In derselben Häufigkeit $(49 \%)$, wie im Spätlatenzstadium die Wassermannsche Reaktion positiv ist, finden sich bei den Sektionen von Syphilitikern syphilitische Prozesse an den inneren Organen oder Residuen von solchen, die klinisch vollkommen latent verliefen ( Fr. Lesser).

2. Der Parallelismus zwischen positiver Wassermannscher Reaktion und klinischen Erscheinungen von Syphilis (Blaschko, Citron, Fr. Lesser u. a.): In den meisten Fällen tritt mit dem Schwinden der syphilitischen Effloreszenzen eine Umwandlung der Reaktion von positiv in negativ ein, und es erfolgt ein Rückschlag in positiv, sobald Rezidive von Syphilis manifest werden.

3. Die Beeinflussung der Reaktion durch antisyphilitische Behandlung: a) Mit fortschreitender antisyphilitischer Kur tritt auch eine fortschreitende Umwandlung der positiven Reaktion in eine negative ein (Fr. Lesser, Höhne, Blaschko). b) Bei Gegenüberstellung von gut und schlecht mit $\mathrm{Hg}$. vorbehandelten Syphilitikern zeigen die gut behandelten im Spätstadium häufiger negative Reaktion als die schlecht behandelten (Citron, Bruck und Stern, Fr. Lesser, Höhne, Pürckhauer etc.).

Das sind im wesentlichen die Tatsachen, die zu dem Schluß berechtigen, daß eine positive Reaktion noch bestehende Syphilis anzeigt. Einen absoluten Beweis stellen die angeführten Argumente zwar nicht dar, dazu wäre es nötig, daß wir die Schlupfwinkel der Spirochäten während des Latenzstadiums kennten und am Sektionsmaterial dann in jedem Falle von positiver Reaktion auch Spirochäten mikroskopisch nachwiesen. Es ist auch richtig, daß die noch unbekannten Stoffe im Blutserum Syphilitischer, welche die positive Reaktion auslösen, nichts Direktes mit den Spirochäten zu tun haben und insbesondere keine Stoffwechselprodukte der Spirochäten darstellen. Es spricht vielmehr alles dafür, daß die Substanz, die die Reaktion auslöst, körpereigen ist, aber im Serum der Syphilitiker in vermehrter Menge kreist. Wir wissen ferner von dem X-Stoff, daß er, was hier nebensächlich ist, eine Affinität zu Lipoiden hat. Die Vermehrung des unbekannten Stoffes steht aber in innigstem Zusammenhang mit der Tätigkeit der Spirochäten, da, wie schon erwähnt, ein Parallelismus zwischen positiv werdender Reaktion und dem Auftreten klinischer Syphilissymptome besteht. Ebenso wie die syphilitische Papel eine lokale Vermehrung von körpereigenen Zellen, d. h. von Leukozyten und Endothelzellen in der Haut darstellt, so stellt sich die positive Reaktion als eine Vermehrung der körpereigenen, zu Lipoiden eine besondere Affinität besitzenden Substanz im Blute dar. Wir können daher noch einen Schritt weiter gehen und die positive Reaktion nicht nur als Zeichen noch bestehender Syphilis, sondern mit Citron direkt als Symptom der Syphilis ansehen, und zwar als ein Symptonl von ganz besonderem klinischen Werte. Im allgemeinen lernen wir in der Frühperiode nur die sichtbaren Symptome an der Haut und den Schleimhäuten kennen, während die an den inneren Organen sich abspielenden syphilitischen Prozesse klinisch latent verlaufen. Daher dokumentiert sich die Syphilis vormehmlich als Hautkrankheit, was sie doch nicht ist. Aber auch in der Spätperiode entgehen, wie ich in früheren Arbeiten zahlenmäßig festgestellt habe, die syphilitischen Prozesse an den inneren Organen meist der klinischen Beobachtung. Sie werden nur dann diagnostiziert, wenn sie Symptome hervorrufen, und dies ist meist der Fall, wenn sie im Zentralnervensystem lokalisiert sind. Durch die Serodiagnostik ist nun hierin Wandel geschaffen worden, indem die positive Reaktion sowohl die klinisch in Erscheinung tretenden als auch klinisch latenten Veränderungen der Syphilis zum Ausdruck bringt. Deshalb ist auch die positive Reaktion, als Symptom betrachtet, das konstanteste aller Syphilissymptome und das am schwersten zum Verschwinden zu bringende Symptom. Aber gerade diese Eigenschaften befähigen die Serumreaktion dazu, als Maßstab für die Therapie zu dienen.

Wenn die positive Reaktion durch eine antisyphilitische Kur in eine negative umgewandelt ist und wir oft später wieder einen Rückschlag in eine positive Reaktion finden, so entspricht dieses Rezidivieren der positiven Reaktion durchaus dem klinischen Verlauf der Syphilis. Wir können das Eintreten von Rezidiven in der Frühperiode der Syphilis beinahe als Regel bezeichnen, wobei noch zu berïcksichtigen ist, daß die Rezidive viel häufiger sind, als sie sich uns klinisch offenbaren.

Wenn man der Ansicht ist, daß eine positive Reaktion nur ein Beweis dafür ist, daß der Betreffende einmal Syphilis gehabt hat und daß trotz positiver Reaktion die Syphilis ausgeheilt sein kann, so müßte man den X Stoff im Blutserum mit Immunstoffen, ähnlich der Vidalschen Reaktion bei Typhus, in Beziehung bringen. Hiernach wäre die positive Reaktion eher als ein Vorteil gegenüber einer negativen Reaktion zu betrachten, und damit steht im Widerspruch die positive Reaktion bei Tabes und Paralyse und ïberhaupt bei manifesten Erscheinungen von Syphilis, sowie der Einfluß der spezifischen Behandlung auf den Ausfall der Reaktion.

Aber wenn auch vom rein theoretischen Standpunkt die Deutung einer positiven Serumreaktion im Sinne noch bestehender Syphilis strittig sein kann, in praktischer Hinsicht kommen ganz andere Gesichtspunkte in Betracht. Nachdem sich gezeigt hat, daß in den meisten Fällen von Tabes (in 112 Fällen $76 \mathrm{mal}$ ) und in sämtlichen Fällen von Paralyse (wir haben 95 Paralytiker serologisch untersucht) die Reaktion positiv ist, so können wir, da an einem engen Zusammenhang dieser - von mir als quartär-syphilitisch bezeichneten Nervenkrankheiten mit der Syphilis nicht zu zweifeln ist, auch umgekehrt schließen, daß Syphilitiker, welche im Spätstadium der Syphilis eine negative Reaktion zeigen, nicht Gefahr laufen, Tabes und Paralyse zu bekommen. Diese Erwägung allein sollte den Praktiker bestimmen, jede Skepsis bezüglich der Beweiskraft einer positiven Reaktion fallen zu lassen und als Ziel einer jeden Syphilisbehandlung eine dauernd negative Reaktion zu erblicken. Die schweren Veränderungen an den inneren Organen in der Spätperiode der Syphilis und unter diesen vornehmlich Gefäßerkrankungen, Tabes und Paralyse sind es, welche die Syphilis zu einer so schweren Krankheit stempeln und welche für die Prognose der Syphilis in erster Linie in Betracht kommen. Wir wollen eben nicht die positive Reaktion an sich bekämpfen, sondern wir wollen sie deshalb bekämpfen, weil uns die positive Reaktion den Eintritt der Tabes und Paralyse fürchten läßt. Der Behauptung von Schuster, Cron, Collins, Nonne, daß die Hg-Behandlung in der Frühperiode keinen Einfluß auf die Verhüitung der Tabes hat, muß ich durchaus widersprechen. Unter den 112 Tabesfällen unseres Laboratoriums hat sich in 2 Fällen die Tabes unmittelbar nach schwerem Trauma entwickelt. In den restierenden 110 Fällen wurde Lues in 28 Fällen negiert. Von diesen 28 Fällen, die demnach nie mit $\mathrm{Hg}$ behandelt waren, reagierten nach Wassermann 24 positiv, d. h. $86 \%$. Von den 82 Tabikern mit positiver Luesanamnese reagierten 52 positiv, d. h. nur $63 \%$. Diese zuerst paradox erscheinende Tatsache, daß Tabiker ohne Luesanamnese weit häufiger positiv reagieren als Tabiker mit Luesanamnese, ist so hervorstechend, daß Citron direkt durch dieses Phänomen auf die Vermutung kam, daß die $\mathrm{Hg}$-Behandlung vielleicht von Einfluß auf die Wassermannsche Reaktion ist und daß das Fehlen der Behandlung bei den die Luesinfektion verneinenden und die erfolgte Behandlung bei den die Infektion bejahenden Tabikern die Ursache der Differenz in dem Ausfall der Serumreaktion war, was ihm auch weitere Untersuchungen bestätigten.

Während wir bei positiver Reaktion, in welchem Stadium der Syphilis sie auch angetroffen wird, stets auf noch vorhandenes Virus schließen, müssen wir bei der Deutung der negativen Reaktion vorsichtiger sein und insbesondere das Alter der bestehenden Syphilis berücksichtigen. Wir müssen die negative Reaktion verschieden beurteilen, je nachdem wir sie im Frühstadium, zu denen wir die ersten vier Jahre nach der Infektion rechnen, oder im Spätstadium der Syphilis antreffen. Wir sehen in den ersten Jahren der Infektion einen häufigen Wechsel der Reaktion von positiv in negativ und von negativ in positiv, und dieser Wandel der Reaktion vollzieht sich häufig spontan, fast konstant aber im Verlauf oder im Gefolge einer antisyphilitischen Behandlung. Er ist wohl als Ausbruch des lebhaften Kampfes zwischen Organismus und Spirochäten an- 
zusehen, in welchem bald der Organismus (negative Reaktion), bald die Spirochäten (positive Reaktion) die Oberhand gewinnen. Die Erfahrung aber zeigt, daß entsprechend dem Alter der Infektion auch ungemein viel seltener die positive Reaktion sich spontan in eine negative umwandelt, anderseits eine nach Quecksilberkur eingetretene negative Umwandlung viel seltener ins Positive zurückschlägt. Dies entspricht ja auch durchaus unseren bisherigen klinischen Erfahrungen, daß mit dem Alter der Infektion auch der Kampf zwischen Syphiliserreger und Organismus ruhigere Formen annimmt. Das Virus schwächt sich mit der Zeit ab, und diese Abschwächung äußert sich klinisçh in der Abnahme der Uebertragbarkeit der Syphilis und in dem selteneren Auftreten von Rezidiven.

Demgemäß läßt eine negative Reaktion im Frühstadium der Syphilis keinerlei Schluß auf die Ausheilung der Krankheit zu, im Spätstadium dagegen darf man mit größter Wahrscheinlichkeit (mit um so größerer Wahrscheinlichkeit, je länger die Infektion und die spezifischen Kuren zurückliegen) auf Ausheilung der Syphilis schließen. Wir glauben, das fünfte Jahr der Infektion als untere Grenze festsetzen zu können, um von $\mathrm{da} a \mathrm{~b}$ aus einer negativen, insbesondere wiederholt negativ befundenen Reaktion mit Wahrscheinlichkeit auf eine Ausheilung der Syphilis schließen zu dürfen.

Es sind nun gegen die therapeutische Bedeutung der Wassermannschen Reaktion verschiedene Einwände erhoben worden, welche wir einer kritischen Würdigung unterziehen wollen.

1. Einwand: Eine nicht geringe Zahl von Fällen reagiert trotz manifester Erscheinungen negativ. Hierzu muß bemerkt werden, daß diese Fälle mit der fortgeschrittenen Technik der Reaktion auf kaum 2\% reduziert worden sind. Besonders das Arbeiten mit wäßrigen Organextrakten und dem von mir angegebenen, jederzeit leicht herstellbaren Aetherextrakt aus normalem Menschenherzen ${ }^{1}$ ) hat zu einer bedeutenden Verfeinerung der Reaktion beigetragen, soda $\beta$, wie gesagt, eine negative Reaktion bei manifesten Syphiliserscheinungen eine Seltenheit geworden ist. Die negative Reaktion ist in diesen Ausnahmefällen zum Teil vielleicht darauf zurückzuführen, daß es sich um rein lokale Rezidive in den obersten Hautschichten handelt, die als abgekapselte Herde daselbst ,,reaktionslos" vegetieren. Wir haben nämlich beobachtet, daß es besonders die ringförmig angeordneten Hautsyphilide sind, die zuweilen keine positive Reaktion auslösen, und gerade die anullären Formationen können als Ausdruck einer serpiginösen, rein lokalen Ausbreitung von abgekapselten Spirochäten in der Haut aufgefaßt werden. In ganz frischen Fällen kommt ferner manchmal ein verspäteter Eintritt der positiven Reaktion zustande, sodaß erst mit dem Ausbruch der Roseola oder gar noch einige Tage später die Reaktion positiv zu werden anfängt.

2. Einwand: Es gibt Fälle von Syphilis, die negativ reagieren und nach Beendigung einer syphilitischen Kur positive Reaktion zeigen. Auch solche Fälle gehören zu den allerseltensten Ausnahmen. Es handelt sich gewöhnlich um Patienten mit ganz frischer Roseola, wo das Blut noch nicht positiv reagiert und, wie eben erwähnt, die reagierende Substanz verspätet (während der Kur) im Blute auftritt. Das Quecksilber hat in diesen frischen Fällen nicht die Kraft, die Produktion der reagierenden Substanz zu verhindern. Den besten Beweis, daß zuweilen bei frischen Fällen von Syphilis das Quecksilber im Kampfe mit dem syphilitischen Virus unterliegt, stellen die Fälle dar, wo gleich zu Beginn des Primäraffektes mit der Quecksilberkur begonnen wird und dennoch bald nach Beendigung der Kur die Roseola hervorbricht. Und haben wir es nicht schon oft erlebt, daß wir im Latenzstadium eine Präventivkur einleiteten und während oder am Schluß der Kur plötzlich syphilitische Effloreszenzen sich zeigten? Schließen wir etwa daraus, daß das Quecksilber gegen Syphilis unwirksam ist?

3. Ein wand: Eine nicht geringe Zahl positiver Reaktionen bleibt trotz ausgiebiger Behandlung positiv. Auch dieser Einwand beweist nur, daß das Quecksilber zuweilen nicht zum

1) Austitrierter wäBriger Organextrakt ist in der Tauentzien-Apotheke, Berlin W. 50 erhältlich. gewünschten Ziele führt und daß es Fälle gibt, welche sich refraktär gegen Quecksilber verhalten. Hier führen zuweilen, wie Mühs a m beobachtet hat, Atoxylininjektionen zur negativen Umwandlung.

4. Einwand: Eine beträchtliche Zahl durch die Behandlung negativ gewordener Fälle springt bald wieder ins Positive um. Die Beobachtungen, auf Grund deren dieser Einwand erhoben wurde, beziehen sich vornehmlich auf Fälle im Frühstadium. Wir haben schon erwähnt, daß dieser Rückschlag im Frühstadium der Syphilis vorkommt und entsprechend dem Alter der Infektion an Häufigkeit abnimmt. Das Rezidivieren der positiven Reaktion entspricht also durchaus dem Rezidivieren der Syphilissymptome im Frühstadium überhaupt, die ja auch entsprechend dem Alter der Infektion an Häufigkeit abnehmen.

5. Einwand: Auch Fälle, die garnicht oder ganz ungenügend in der Frühperiode mit Quecksilber behandelt worden sind, zeigen später negative Reaktion. Es besteht kein Grund, solche Fälle gegen die therapeutische Bedeutung der Wassermannschen Reaktion ins Feld zu führen. Die Fälle zeigen vielmehr, daß das Quecksilber nicht das alleinige Heilmittel für die Syphilis ist, sondern daß auch eine natürliche Ausheilung der Syphilis vorkommen kann. Wir kommen auf diesen Punkt später noch zurück.

Wenn wir uns Rechenschaft geben, was für Erfolge die bisher geläufigen Behandlungsmethoden der Syphilis gezeitigt haben, so finden wir das Lager der Syphilidologen in die Anhänger der symptomatischen-exspektativen Behandlung und in die der chronisch-intermittierenden, präventiven Behandlung geteilt. Jede Partei hat, auf reine Empirie fußend, mit ihrer Methode gute Resultate. Welcher Methode der Vorzug zu geben ist, darüber liegen einwandfreie Erhebungen nicht vor, denn allen diesbezüglichen Statistiken haftet der Fehler an, daß sie immer von den Tertiärsyphilitikern ausgehen, aber die Syphilitiker unberücksichtigt lassen, die nicht tertiär werden und darum im allgemeinen unseren Beobachtungen entschwinden. Es wird z. B. gezeigt, daß die meisten Syphilitiker mit Tertiärerscheinungen wenig Kuren in der Frühperiode durchgemacht haben, und daraus wird dann immer der Trugschluß gezogen: je weniger Kuren, um so häufiger Tertiärerscheinungen. Man übersieht dabei, daß eben die meisten Syphilitiker wenig Kuren durchmachen und nur wenige Syphilitiker sich zu vier und mehr Kuren entschließen. Es muß also heißen: Je weniger Kuren, um so mehr Syphilitiker, folglich auch um so mehr Tertiärsyphilitiker. Es geht aber daraus kein Kausalnexus zwischen schlechter $\mathrm{Hg}$-Behandlung und Tertiärerscheinungen hervor. Auch die oft zitierte Tabesstatistik von Schuster, Cron etc. ist auf diesem Trugschluß aufgebaut. Es komnt nicht darauf an, wieviel Prozent von den Tabikern schlechte Kuren gemacht haben, sondern wieviel Prozent von schlecht behandelten Syphilitikern später Tabiker werden. Die Serodiagnostik gestattet nun, dem erwähnten Trugschlusse zu begegnen, und ermöglicht, einwandfreie Statistiken aufzustellen. Wenn wir nämlich bei allen den Syphilitikern, deren Infektion mindestens fünf Jahre zurückliegt und die keinerlei klinische Symptome von Syphilis mehr aufweisen, prüfen, wie häufig sich eine positive bzw. negative Reaktion findet, wenn früher

\begin{tabular}{|c|c|c|c|c|}
\hline $\begin{array}{c}\text { Anzahl } \\
\text { früherer Kuren }\end{array}$ & $\begin{array}{c}\text { Anzahl } \\
\text { der Fälle }\end{array}$ & + & - & $-\%$ \\
\hline $\begin{array}{c}\text { keine } \\
1 \\
2 \\
3 \\
4 \\
5 \\
6\end{array}$ & $\begin{array}{r}33 \\
123 \\
84 \\
140 \\
57 \\
34 \\
28\end{array}$ & $\begin{array}{l}28 \\
75 \\
42 \\
62 \\
26 \\
12 \\
13\end{array}$ & $\begin{array}{r}5 \\
48 \\
42 \\
78 \\
31 \\
22 \\
15\end{array}$ & $\begin{array}{l}15 \\
39 \\
50 \\
56 \\
54 \\
65 \\
53,5\end{array}$ \\
\hline $\begin{array}{r}7 \\
8 \\
9 \\
10 \\
11 \\
12 \\
13 \\
14\end{array}$ & $\begin{array}{r}9 \\
8 \\
3 \\
3 \\
- \\
\mathbf{1} \\
\mathbf{1} \\
\mathbf{1}\end{array}$ & $\begin{array}{c}4 \\
3 \\
1 \\
\mathbf{1} \\
- \\
\mathbf{1} \\
\end{array}$ & $\begin{array}{r}5 \\
5 \\
2 \\
2 \\
1 \\
1\end{array}$ & $\begin{array}{l}\text { Die Anzabl der Fälle } \\
\text { ist zu gering, um nach } \\
\text { Prozenten umgerechnet } \\
\text { zu werden. }\end{array}$ \\
\hline $\begin{array}{l}5 \text { und mehr } \\
6 \text { und mehr } \\
7 \text { und mehr }\end{array}$ & $\begin{array}{l}88 \\
54 \\
26\end{array}$ & $\begin{array}{l}35 \\
23 \\
10\end{array}$ & $\begin{array}{l}53 \\
31 \\
16\end{array}$ & $\begin{array}{l}60 \\
57 \\
61\end{array}$ \\
\hline Summa & 525 & 268 & 257 & 49 \\
\hline
\end{tabular}


gar keine Kur, wie häufig, wenn eine, zwei, drei, vier und mehr Kuren durchgemacht worden sind, so ergibt sich folgende Statistik, die auf 525 Fällen aufgebaut ist, bei denen das Jahr der Infektion und die Anzahl der früheren Kuren genau vermerkt ist.

Vorstehende Statistik läßt folgende sehr bemerkenswerte Schlüsse z11:

1. Die Syphilis kommt nur in der Hälfte aller Fälle (49\%) zur Ausheilung.

2. Es gelingt in $39 \%$, durch eine einzige $\mathrm{Kur}$ in der Frühperiode die Syphilis später zur Ausheilung zu bringen.

3. Die Abnahme der positiven bzw. Zunalıme der negativen Reaktion geht proportional der Anzahl früherer Kuren, jedoch mit der Einschränkung, daß bei vier Kuren der Höhepunkt (55-65\% Ausheilung) erreicht wird.

4. Garnicht mit Hg behandelte Syphilitiker zeigen nur selten $(15 \%)$ negative Reaktion.

ad 1. Daß nur bei der Hälfte aller Infizierten die Syphilis wirklich zur Ausheilung kommt, wird vielleicht die meisten Kliniker überraschen und als nicht zutreffend erscheinen. Man muß sich immer wieder vergegenwärtigen, daß sich die Nichtausheilung der Syphilis sehr häufig klinisch nicht dokumentiert, während die positive Reaktion das einzige nachweisbare Symptom bleibt, welches viele Syphilitiker, die an irgendeiner interkurrenten Krankheit sterben, mit ins Grab nehmen. Aber wenn auch die positive Reaktion nichts über die Schwere, Ausdehnung und Lokalisation der latenten Syphilis aussagt, so muß man doch anderseits in Betracht ziehen, daß Lente mit positiver Reaktion jederzeit ein Rezidiv der Haut, aber auch schwerere Schädigungen wie Aortenaneurysma, Tabes und Paralyse bekommen können.

ad 2. Daß es so häufig (in $39 \%$ ) gelingt, dırch eine einzige Kır eine Ausheilung der Syphilis herbeizuführen, wird allgemein ïberraschen. Wir dïrfen natürlich nicht annehmen, daß unmittelbar nach der einmaligen Kur eine Abortivheilıng der Syphilis erfolgt ist. Dem widerspricht die Tatsache, daß in den meisten Fällen die durch die erste Kur negativ gewordene Reaktion spätel wieder in positiv umschlägt. Anclı die Statistik lehrt, daß in $74 \%$ die Reaktion im Beginn des zweiten Jahres nach der Infektion positiv ist.

ad 3. Wir finden zum ersten Male eine einwandfreie Bestätigung für die Behauptung, welche die Anhänger der chronisch-intermittierenden Behandlung von jeher vertreten haben, daß das Quecksilber in der Frühperiode nicht bloß die Symptome beseitigt, sondern daß es auf die Spirochäten selbst lähmend wirkt, bzw. daß es den Organismus im Kampf mit den Spirochäten unterstützt. Das geht anch recht einleuchtend ans folgender Statistik hervor, in welclier das Alter der Syphilis berücksichtigt ist :

\begin{tabular}{|c|c|c|c|c|c|c|c|c|c|}
\hline \multirow{3}{*}{$\begin{array}{c}\text { Anzah1 } \\
\text { früherer } \\
\text { Kuren }\end{array}$} & \multicolumn{6}{|c|}{ Anzah 1 der Fălle } & \multicolumn{3}{|c|}{ Ausgedrūckt in $\%$ negativ: } \\
\hline & \multicolumn{2}{|c|}{$\begin{array}{l}5-9 \text { Jahre } \\
\text { nach der } \\
\text { Infektion }\end{array}$} & \multicolumn{2}{|c|}{$\begin{array}{l}\text { 10-19 Jahre } \\
\text { nach der } \\
\text { Infektion }\end{array}$} & \multicolumn{2}{|c|}{$\mid \begin{array}{c}20-40 \text { Jahre } \\
\text { nach der } \\
\text { Infektion }\end{array}$} & \multirow{2}{*}{$\begin{array}{c}5-9 \text { Jahre } \\
\text { nach der } \\
\text { lnfektion } \\
- \\
\end{array}$} & \multirow{2}{*}{$\begin{array}{c}10-19 \text { Jahre } \\
\text { nach der } \\
\text { Infektion } \\
-\end{array}$} & \multirow{2}{*}{$\begin{array}{c}20-40 \text { Jahre } \\
\text { nach der } \\
\text { Infektion } \\
-\end{array}$} \\
\hline & + & & + & 一 & \pm & . & & & \\
\hline 1 un & & & & & & & $\%$ & $\%$ & $\%$ \\
\hline $\begin{array}{l}\text { Kuren } \\
3 \text { Kuren }\end{array}$ & $\begin{array}{l}40 \\
27\end{array}$ & 29 & 54 & 40 & 22 & 22 & 42 & 42,5 & 50 \\
\hline 4 u. mehr & 39 & & & & & & & & \\
\hline & 39 & 39 & 18 & 33 & 4 & 12 & 50 & 65 & $\left.(75)^{1}\right)$ \\
\hline
\end{tabular}

Wir sehen aus vorstehenden Zahlen, daß bei den Syphilitikern, welche in der Frühperiodeviele Kurendurchgemacht haben, mit zunehmendem Alter der Syphilis die negative Umwandlung viel häufiger eintritt als bei Syphilitikern, die nur eine oder zwei Kuren durchgemacht haben. Die natürliche Ausheilung der Syphilis hat bisher m. E. viel zu wenig Beachtung gefunden, und der Grund hierfür ist wohl darin zu suchen, daß wir niemals den Beweis

1) Diese Prozentzahl kann wegen der geringen Anzahl von 16 Fällen nicht einwandfrei verwertet werden. für die wirkliche Ausheilung erbringen konnten. Erst die Serodiagnostik ermöglicht einen sicheren Beweis und einen zahlenmäßigen Beleg für die Selbstreinigung des Organismus vom syphilitischen Virus. Wir wußten allerdings längst, daß mit der Daner der Infektion eine Abschwächung des syphilitischen Virus eintritt und daß allgemeinere Ausbrüche der Krankheit, entsprechend dem Alter der Syphilis, seltener werden. Die Abschwächung des syphilitischen Virus mit dem Alter der Infektion kannten wir ja auch aus der Vererbung, aus der Deszendenz. Welches nun die Hilfsmittel sind, die der Organismus, vom Quecksilber abgesehen, zur Selbstreinigung vom syphilitischen Virus besitzt und wie sich ihre Wirkung äußert, davon wissen wir nichts. Ihre Kenntnis wäre für die Behandlung der Syphilis von eminenter Bedeutung, um möglicherweise dann den Organismus im Kampfe gegen die Spirochäten durch geeignete Maßnahmen zu unterstiitzen. Daß aber natïrliche Schutzkräfte häufig zu einer völligen Ausheilung der Syphilis führen, das sehen wir unter anderem auch aus dem verschiedenen Verlauf der Syphilis bei Eheleuten, wo man trotz gleicher Behandlung bei dem einen Ehegatten in der Spätperiode eine negative Reaktion findet, während der andere Ehegatte dauernd positiv reagiert. Anderseits aber wird durch die chronisch-intermittierende Behandlung in nicht mehr als $65 \%$ der Fälle eine negative Reaktion erzielt. Wir müssen daher zugeben, daß diese Behandlungsart doch wohl in ihrer Heilwirkung überschätzt worden ist und nicht das erreicht, was man von ihr, auf reiner Enipirie fußend, vorausgesetzt hat. Nachdem die Statistik gezeigt hat, daß wir durch acht Kuren in der Frülıperiode nicht viel mehr erreichen als durch drei bis vier Präventivkıren und daß die Ausheilung der Syphilis zwar proportional der Anzahl der $\mathrm{K}_{u}$ iren geht, dabei aber eine gewisse Grenze nicht ïberschreitet, miissen wir es als unzweckmäßig und nutzlos bezeichnen, für alle Fälle bedingungslos acht Kuren vorzuschreiben. Ja, es ist sogar zu erwägen, ob überhaupt eine Präventivbehandlung bedingungslos fïr alle Fälle während der Frïhperiode indiziert ist, da wir doch in der nicht zu interschätzenden Häufigkeit von $39 \%$ durch eine einmalige Kur eine Ausheilung erzielen, sodaß also Präventivkuren einerseits in $39 \%$ unnötigerweise gemacht werden, anderseits durch die Präventivkuren nur in $60 \%$, also in nur weiteren $21 \%$ eine Ausheilung erzielt wird. Wir möchten uns aber doch in jedem Falle fïr die Durchfïhrung von drei bis vier Kuren entscheiden, zumal in der Praxis schon die Häufigkeit der Rezidive zu mehreren, sagen wir zwei bis vier Kuren, zwingt, also die symptonatisch-exspektative Behandlung sich der intermittierenden präventiven Behandlıng sehr nähert und ihr in vielen Fällen sogar gleichkommt, wofern wir nur nicht die ungeheure Menge von acht Kuren einer Präventivbelıandlung bedingungslos zugrunde legen.

ad 4. Einer besonderen kurzen Besprechung wert erscheinen uns die Fälle von Syphilis, die überhaupt nie mit Quecksilber behandelt worden sind. Es ist erstaunlicl, wie giinstig der Einfluß einer einzigen $\mathrm{Hg}-\mathrm{Kur}$ ist, wenn man dagegen die Fälle betrachtet, die niemals mit $\mathrm{Hg}$ behandelt worden sind und die in nur $15^{\circ}$ später zn einer negativen Reaktion führen. Die Zahl der garnicht mit $\mathrm{Hg}$ behandelten Syphilitiker muß sehr groß sein, da ungemein häıfig die Syphilis zur Zeit der Infektion dem Träger garnicht zum Bewußtsein kommt und sich erst dokumentiert, wenn in der Spätperiode Tertiärerscheinungen, Tabes oder Paralyse, hervorbrechen. Von 247 Fällen, die mit Tertiärerscheinungen der Hant die Klinik aufsuchten, wurde $87 \mathrm{mal}, \mathrm{d}$. h. in $35 \%$, Lues anamnestisch negiert. Ebenso wiesen, wie schon erwähnt, unter 112 Tabesfällen 30 eine negative Anamnese anf, und unter diesen Tabikern mit negativer Anamnese war die Serumreaktion, wie eingangs erwähnt, in 24 Fällen positiv. Wenn sich auch hiernach der Gedanke aufdrängt, daß das Fehlen jeder spezifischen Behandlung häufiger zu Tabes oder Tertiärerscheinungen führe, so wäre doch ein Schluß in dieser Richtung voreilig, da wir ja garnicht die Anzahl der Syphilitiker kennen, die sich ihrer Krankheit nicht bewußt sind und auch später weder Tertiärerscheinungen noch Tabes bekommen.

Pürckhauer hat an dem Material der Neisserschen Klinik eine Statistik aufgestellt, die mit der meinigen Differenzen aufweist und für 
die spätlatenten Syphilitiker unter Berücksichtigung der Anzahll der Kuren folgende Zahlen ergibt:

\begin{tabular}{c|c|c|c}
\hline Kuren & + & - & $-\%$ \\
\hline $\mathbf{l}$ & 19 & $\mathbf{1 9}$ & $\left.50^{1}\right)$ \\
2. & 9 & 21 & 70 \\
3. & 13 & 24 & 65 \\
4. & 7 & 22 & 76 \\
5. & 6 & 24 & 80 \\
6. & 6 & 33 & 85 \\
7. & 6 & 25 & 80 \\
8. & 3 & 30 & 90
\end{tabular}

Hierzı möchte ich bemerken, daß Pürckhauer das Spätlatenzstadium schon vom Beginn des dritten Jahres der Infektion an gerechnet hat und daß demgemäß bei Patienten mit fünf, sechs, sieben und acht Kuren in einem Teil der Fälle eine direkte Beeinflussung der Reaktion durch die letzte, noch nicht lange genug zurückliegende $\mathrm{Hg}$-Kur nicht. ausgeschlossen erscheint. Für die Differenz in dem Ergebnis der Statistiken kommt ferner die Anwendung verschiedener Arten Organextrakte in Betracht, da von Pürcklıauer der alkolıolische, von mir der empfindlichere und mehr positive Reaktionell gebende, wäBrige Organ. extrakt benutzt wurde. Ferner hat Pürckhauer nur bei totaler Hemmung der Hämolyse die Reaktion als positiv bezeichnet, während ich auch die Fälle mit Kuppe als positiv eraclitet habe. So ist es viel. leicht zu erklären, daß sich bei Pürckhauer schon nach einer einzigen Kur 50\% (bei mir nur 39\%) negativer Reaktion ergeben. Wir finden ferner bei Pürckhauer eine, wenn auch nicht gleichmäßige Zunahme der negativen Reaktion auch nach mehr als vier Kuren, jedoch erscheint mir vom praktischen Gesichtspunkt die Differenz in den Erfolgen beispielsweise zwischen vier und sieben Kuren (76:80\%) zu gering, um jeden Patienten bedingungslos mit so vielen Kuren zu belasten.

Können wir nun durch eine geeignetere Anordnung oder durch eine rationellere Verteilung der antisyphilitischen Kuren bessere Resultate erzielen und mehr als nur $60-65 \%$ der Syphilitiker einer dauernd negativen Reaktion zuführen?

Zunächst müssen wir uns gegen die schablonenhafte Festlegung von 30 Einreibungen oder 15 unlöslichen oder 30 löslichen $\mathrm{Hg}$-Injektionen für eine Kur wenden. Was man unter einer guten Kur zu verstehen hat, darf nicht von der Menge des einverleibten Medikaments, sondern von dessen Wirkung bei dem betreffenden Individuum abhängen. Auch hierin ist ein enormer Fortschritt durch die Serodiagnostik zu verzeichnen, da diese uns nicht nur anzeigt, ob wir behandeln nü̈ssen, sondern auch, wie lange wir die begonnene Kur fortzusetzen haben. Ich habe als erster auf die direkte Beeinflussung der Wasser mannschen Reaktion durch die $\mathrm{Hg}$-Kur hingewiesen und gezeigt, daß es fast in jedem Falle gelingt, die positive Reaktion durch eine genügend energische Kur in eine negative umzuwandeln, und daß die dazu notwendige $\mathrm{Hg}$-Menge individuell verschieden ist. Bei dem einen Patienten genügen zur negativen Umwandlung $\mathrm{z}$. $\mathrm{B}$. 15 Sublimatinjektionen, ein anderer benötigt 30 , wieder ein anderer 40 Injektionen dazu. Bei der Schmierkur spielt auch noch die individuell verschiedene Aufnahmefähigkeit der Haut eine Rolle, und es ist kein Zweifel, dal3 wir, wenn wir ganz schablonenmäßig eine bestimmte Menge $\mathrm{Hg}$ für eine Kur festsetzen, häufig in der Wirkung auf halbem Wege stehen bleiben und dadurch den Erfolg der Kur überhaupt in Frage stellen. Wir müssen uns eben immer wieder klar machen, daß die Schutzkräfte des Organismus im Kampfe mit den Spirochäten eine große, ja vielleicht die größte Rolle spielen und daß diese Schutzkräfte in jedem Organismus verschieden stark gebildet werden. Die Wasser mannsche Reaktion ist besonders insofern geeignet als Maßstab für die notwendige Dauer der einzelnen Kur zu dienen, als die positive Reaktion, wie nochmals betont sei, ein Indikator für alle Symptome sowohl für die klinisch in Erscheinung tretenden, als auch für die klinisch latent verlaufenden ist und die Beseitigung eines jeden Symptoms mit dem Negativwerden der Reaktion Hand in Hand geht. Die spezifische Kur ist demnach so lange fortzusetzen, bis eine negative Reaktion erreicht ist, wofern dies, ohne $\mathrm{zu}$ forcieren, möglich ist. Schädigungen habe ich durch energischere Kuren niemals gesehen, und es ist ja nur selbstverständlich, obwohl manche Autoren etwas ganz Besonderes damit zu sagen glauben, daß man sich der Individualität des Patienten vollkommen anpassen

1) Die Prozentverhältnisse sind von mir hinzugefúgt worden. muß. Es ist aber auch kein Zweifel, daß die Quecksilberkuren in Aachen, Nenndorf etc. viel energischer und intensiver, als allgemein üblich, bei besten Wohlbefinden der Patienten durchgefïhrt werden.

Ich nehme die serologische Untersuchung unmittelbar nach Beendigung der Kur vor und kann Citron nicht beipflichten, daß die serologische Untersuchung erst vier bis sechs bis acht Wochen nach Beendigung der Kur einen wirklichen Wert habe, da unmittelbar nach Abschluß der Behandlung die Reaktion noch positiv sein und die Syphilis dabei doch geheilt sein könne, indem die Zellen, die die Antikörper produzieren, nicht sofort mit der Produktion sistierten und auch die Ausscheidung der Antikörper eine gewisse Zeit erfordere. Diese theoretischen Voraussetzungen treffen nach meinen Untersuchungen in praxi nicht zu. Ich habe bei einer Anzahl Patienten, die nach Beendigung der Kur nur noch schwach positiv reagierten, die Behandlung abgebrochen und deren Blut nach vierwöchentlicher Pause wieder untersucht, und siehe da: die schwach positive Reaktion war jetzt wieder stärker positiv geworden. Bei der praktischen Durchführung läuft die Differenz zwischen Citrons und meinem Vorschlage darauf hinaus, daß ich noch etwas länger behandle, nach Citrons Ansicht über die negative Umwandlung hinaus. Es entspricht dies durchaus der Gepflogenheit, die wir auch bei allen übrigen Symptomen der Syphilis befolgen, d. h. wir behandeln weiter, wenn auch die Symptome längst geschwunden sind, und ich finde eigentlich einen Widerspruch in dem Vorschlage Citrons, wenn er die positive Reaktion als Symptom der Syphilis auffaßt, dieses Symptom aber anders therapeutisch behandelt haben will als alle anderen Symptome der Syphilis. Alles in allem kann ich also nur einen Vorteil darin erblicken, wenn man verlangt, daß zugleich mit Beendigung der Kur auch eine negative Reaktion vorliegt. Wir haben allen Grund zu hoffen, daß wir durch solche individuell angepaßten Kuren bessere Resultate bei der Behandlung der Syphilis als bisher erzielen werden.

Auch ein weiterer Weg scheint nuir noch gangbar zu sein und für die Fälle in Betracht zu kommen, bei denen wir durch drei bis vier in der Frühperiode ausgeführte und bis zur negativen Umwandlung fortgesetzte Präventivkuren nicht zum Ziele gelangt sind.

Wir haben gesehen, daß das Quecksilber meist nicht imstande ist, eine Abortivheilung der Syphilis herbeizuführen; das Quecksilber vermag scheinbar die Syphiliserreger nur zu lähmen und ihre Aktivität eine Zeitlang hintanzuhalten. Wir wissen ferner, daß die Spirochäten entsprechend dem Alter der Syphilis an Menge und Virulenz abnehmen, sodaß fünf Jahre nach der Infektion bei der Heirat fast niemals eine Uebertragung auf die Ehegattin oder die Geburt syphilitischer Kinder erfolgt. Wir haben ferner gesehen, daß auch serologisch in dem Einfluß des Quecksilbers auf die Wassermannsche Reaktion mit dem Alter der Infektion eine Aenderung eintritt, indem etwa vom fünften Jahre der Infektion ab ein Rückschlag der negativen Reaktion in eine positive seltener vorkommt. Es bietet somit das im Spätstadium schon geschwächte Virus dem Quecksilber einen günstigeren Angriffspunkt, und es erscheint daher rationell, eine füntte, eventuell auch sechste Kur für die wenigen Fälle zu reservieren, die im Spätstadium noch eine positive Reaktion zeigen. Es ist zu erhoffen, daß wir auf diese Weise dahin kommen werden, weit mehr Syphilitikern zu einer dauernd negativen Reaktion zu verhelfen und damit die Häufigkeit der Tabes, Paralyse etc. herabzusetzen.

Es ist der Vorwurf erhoben worden, daß durch die wiederholten Kuren auf Grund positiver Serumreaktionen Syphilidophoben und Neurastheniker gezüchtet werden. Diese Zustände werden meist durch den $Z$ weifel, ob die Krankheit ausgeheilt ist, erzeugt, und diesem $Z$ weifel begegnet gerade die Serodiagnostik, die bei negativem Ausfall der Reaktion den Patienten von seiner Furcht befreit, bei positivem Ausfall anderseits die Notwendigkeit weiterer Kuren rechtfertigt. Wir glauben, daß der Gewinn für die Kranken weit größer ist als ein eventueller Schaden, und letzterer kann durch Taktgefühl und Geschicklichkeit des Arztes erheblich gemindert werden.

Resümee. Die Entdeckungen der Uebertragbarkeit der Syphilis auf Tiere und des Syphiliserregers sind zurzeit für die 
Therapie der menschlichen Syphilis bedeutungslos. Indessen bietet die Wassermannsche Reaktion eine Richtschnur für die Behandlung, insofern sie uns anzeigt, wie lange wir die Kur auszudehnen und wann wir sie zu wiederholen haben. Die positive Serumreaktion beweist stets die Anwesenheit noch aktiver Spirochäten. Die negative Reaktion läßt im Frühstadium keinerlei Schlïsse zu; in Spätstadium kann man mit einer proportional dem. Alter der Syphilis sich steigernden Wahrscheinlichkeit aus der negativen, besonders der wiederholt negativ befundenen Reaktion auf Ausheilung der Syphilis schließen. Wir finden im Spätstadium häufiger negative Reaktion (Ausheilung), wenn mehrere Hg-kuren im Frühstadium stattgefunden haben, jedoch mit der Finschränkung, daß bei vier Kuren das Maximum der Heilwirkung erreicht wurde. Für die Dauer der Kur darf nicht die $\mathrm{Hg}$-menge sondern die Wirkung (negative Umwandlung) maßgebend sein. Das Ziel jeder Syphilisbehandlung muß die Erreichung einer dauernd negativen Reaktion sein. 